\title{
Pectobacterium atrosepticum plasmid pPA21A is an important determinant of plant-bacterium pathosystem development Diubo Yu. ${ }^{1}$, Nikolaichik Ye. ${ }^{2}$
}

Belarusian State University, Minsk, Belarus

E-mail: yuliyadiubo@gmail.com

Key message. Pectobacterium atrosepticum strain 21A can cause a strong hypersensitive reaction in Nicotiana tabacum. The pPA21A plasmid was found to be responsible for this phenotype. The plasmid genes involved are been identified.

Keywords: plasmid-dependent virulence, Pectobacterium atrosepticum, bacterium-plant interaction

P. atrosepticum is an economically significant plant pathogen. It causes soft rot disease of potato tubers and blackleg of the stems. Most $P$. atrosepticum strains can not cause a hypersensitive reaction in $N$. tabacum, but the $21 \mathrm{~A}$ strain can. It is also more virulent than other $P$. atrosepticum strains from our collection - SCRI1043 and PB72. Strain 21A chromosome has 98,7\% and 99,9\% identity to SCRI1043 and PB72 chromosomes. The major difference between the genomes of these strains is the pPA21A plasmid present in the 21A strain. Since plasmid involvement in the control of Pectobacterium sp. virulence wasn't studied so far, in this work we try to determine if the pPA21A plasmid is responsible for the unique properties of strain $21 \mathrm{~A}$.

The main purpose of the study is the characterization of plasmid pPA21A and the identification of its role in bacterium-plant interaction.

Deletion variants of plasmid pPA21A were constructed by the conventional molecular biology methods. Sirtuin gene and gene of phospholipase D were cloned in the high copy number vector pK18. Testing the properties of the deletion variants of pPA21A was performed with regular microbiology methods. Plant-bacterial interactions were tested by the infiltration of bacterial suspension into Nicotiana tabacum leaves and by injection of the suspensions into potato tubers followed by weighting rotten tuber tissues.

To simplify control of pPA21A presence, a gentamycin resistance gene was added to the plasmid and all further manipulations were performed with pPA21A::Gm.

Conjugative transfer of pPA21A::Gm into plasmid-free strains SCRI1043 and PB72 made these two strains much better inducers of hypersensitive reaction $(\mathrm{HR})$ and also improved their ability to macerate potato tubers. Analysis of pPA21A nucleotide sequence revealed three loci that could be involved in the interaction with plants: a vir-cluster of the type IV secretion system genes and genes coding for a phospholipase D ( pld) and a sirtuin-like protein (sir). To check which of the three loci is responsible for the HR-inducing phenotype, we have constructed deletions of pPA21A::Gm and also cloned the pld and sir genes using a high copy number plasmid. Virulence tests performed with $P$. atrosepticum strains carrying the deletion derivatives of pPA21A have so far shown that (i) vir cluster is required for conjugative transfer of pPA21A, but doesn't contribute to virulence directly and (ii) either the pld or the sir gene is the determinant responsible for HR induction. To identify HR-inducing plasmid determinant more precisely, we are currently characterising $P$. atrosepticum strains with single sir or pld genes introduced on a multicopy plasmid and will present the results at the conference. 